\title{
Systemic vasculitis: a difficult diagnosis
}

$\mathrm{N}$ othing triggers an internist's diagnostic interests more than a potential case of systemic vasculitis. The ubiquitous nature of the target tissue, the variations in the rate and severity of disease progression, and its ability to mimic other diseases mandate an intense and focused approach to diagnosis and treatment. Blood vessels have limited ways of responding to injury: increased vascular permeability, weakening of vessel wall with resultant aneurysm formation or hemorrhage or both, and intimal proliferation and thrombosis which can result in obstruction and ischemia. ${ }^{1}$

Vasculitis is a heterogeneous group of disorders characterized by inflammation, often with necrosis and occlusion of blood vessels. These disorders may be generalized or localized, primary (i.e., the essential disorder affecting blood vessels) or secondary (associated with a variety of different underlyng diseases). The clinical spectrum of vasculitis represents one of the most interesting and perplexing group of diseases in internal medicine. ${ }^{2}$

Since the first classification of vasculitis by Zeek in $1952,{ }^{3}$ many other classification systems have been proposed, ${ }^{47}$ but the lack of pathognomonic clinical and laboratory features, uniform histopathological expression, and unknown or incompletely understood etiology and pathogenesis for the majority of the disorders make it

\section{Address for correspondence:}

Emília Inoue Sato

Rua Botucatu, 740 -Vila Clementino

São Paulo/SP - Brasil - 04023-062 virtually impossible to achieve one classification with universal acceptance. ${ }^{6}$

In the Rheumatology Division of the Universidade Federal de São Paulo (UNIFESP), we use Lie's classification 6 due to its practicality, although we have implemented some minor modifications (Table I). Lie's classification is based on the predominant size of the blood vessels involved and allows for overlap among major vasculitic syndromes.

Several mechanisms of vascular damage have been proposed, including pathogenic immune complex deposition, antiendothelial cell antibody-mediated vessel damage, injuries related to antibodies against lysosomal enzymes (ANCA), cellular immune response and granuloma formation, vessel damage mediated directly by infectious agents, and tumor-cell-mediated vessel damage. Although the role of each one of these mechanisms is variable in any given vasculitis syndrome, in general the lesion is ultimately caused by immune complex deposition, complement system activation, and inflammatory response. The involved antigens include autoantigens (nuclear or cytoplasmatic antigens), infectious antigens (hepatitis B or $C$ virus), tumoral antigens, drugs, and others not yet identified. $^{8}$

The role of adhesion molecules like ICAM-1 and VCAM-1, and inflammatory cytokines such as IL-1, IL-6 and TNF-alfa appear to be important in the pathogenesis of vascular damage in vasculitic syndromes. The expression of these factors in endothelial cells in response to a given initial stimulus (unknown in the majority of cases), may be important in the modulation of the 
Table 1

Classifications of vasculitis. Lie 6 Modified UNIFESP Primary vasculitis

\author{
Vasculitis of large-sized blood vessel \\ Giant cell (temporal) arteritis \\ Takayasu arteritis

\section{Vasculitis of medium- and small-sized blood vessel Polyarteritis nodosa \\ Churg-Strauss syndrome \\ Wegener's granulomatosis \\ Kawasaki disease}

\section{Vasculitis affecting predominantly small-sized blood vessels \\ Microscopic polyangiitis \\ Henoch-Schonlein Syndrome \\ Essential cryoglobulinemic vasculitis \\ Cutaneous leukocytoclastic angiitis}

\section{Miscellaneous conditions \\ Buerger's disease \\ Cogan Syndrome \\ Behçet disease}

\section{Secondary vasculitis \\ Infection-related vasculitis \\ Vasculitis secondary to connective tissue disease \\ Drug-hypersensitivity-related vasculitis \\ Malignancy-related vasculitis \\ Hypocomplementemic urticarial vasculitis \\ Post-organ-transplant vasculitis}

immunological response at the target to the vascular component leading to vascular damage. ${ }^{8}$

Clinical manifestations are polymorphic and depend on the size of the involved vessels, and the localization and extension of the vascular injury. The polymorphism of clinical features frequently brings these patients to a host of different medical specialists. Thus, these patients may be seen by neurologists, cardiologists, nephrologists, vascular surgeons, rheumatologists etc., making the study of vasculitis of great interest to all physicians independent of the speciality.

The most frequent type of vasculitis affects predominantly arterioles, capillaries, and venules, and the corresponding clinical features are mainly skin lesions such as vascular purpura, vesicles or sometimes urticarial lesions. Less frequently, arthritis, glomerulonephritis, abdominal pain, intestinal bleeding and peripheral neuropathy are observed. Systemic complaints are infrequent, except when due to associated diseases (secondary vasculitis syndromes). Laboratory tests are not specific, but may be helpful to diagnose secondary vasculitis such as those associated with autoimmune rheumatic diseases. In all cases of vasculitis affecting mainly small vessels, it is important to look for cryoglobulins; when positive, it is necessary to investigate if it is associated with infections such as B or C hepatitis virus, neoplasm or autoimmune rheumatic diseases. Many different diseases may present vasculitis affecting arterioles, capillaries and venules, thus requiring several laboratory tests for an exact diagnosis.

Small- and medium-size artery involvement is characterized by cutaneous ulceration, distal gangrene, mononeuritis multiplex, hypertension, coronary insufficiency, arthralgia/arthritis, central nervous system involvement, and constitutional symptoms such as weight loss, fever and weakness. Polyarteritis nodosa is the most representative of this group of vasculitis. The presence of asthma and eosinophilia should bring to mind the possibility of Churg-Strauss disease, and the involvement of the upper airways with destructive lesions and pulmonary and/or renal involvement may suggest Wegener's disease. The presence of antineutrophil antibody with cytoplasmic fluorescence pattern (cANCA) supports the diagnosis of Wegener's disease, while the presence of antineutrophil antibody with perinuclear fluorescence pattern (pANCA) is more frequently found in microscopic polyangitis, but is nonspecific and may be found with less frequency in other diseases such as polyarteritis nodosa and systemic lupus erythematosus. ${ }^{9}$

When the involvement is predominantly of largesized arteries, the main clinical features are limb claudication (mostly upper limb), hypertension, neurological symptoms (headache, syncope, stroke, dizziness, vision complaints, transient ischemic attacks) and cardiac involvement. A decreased or absent pulse in young patients reguires arteriography to investigate the possible diagnosis of Takayasu's arteritis. Elderly patients complaining of severe headache should be considered for the diagnosis of temporal arteritis, whiching requires a biopsy of the affected temporal artery. Laboratory tests are not specific, and in the majority of cases, acute phase reactants are raised.

Patient histolosy and clinical examination are extremely important in cases of systemic vasculitis, bringing immediate and valuable information, such as whether the vasculitis is primary or secondary, the size of vessels predominantly affected, and a possible diagnosis hypothesis. The confirmation of vasculitis occurs through the histopathological study of the affected organ and/or 
by arteriography. However, determining the exact cause of vasculitis or the precise classification requires a battery of laboratory tests in accordance with the suspected diagnosis. In some cases, it is not possible to come up with the exact classification of the vasculitis despite all laboratory, histologic and arteriographic examinations.

At the Multiprofessional Vasculitis Clinic at UNEFESP, comprised of rheumatologists, vascular surgeons, and physical therapistis, we studied 69 consecutive patients with systemic vasculitis: 49 ( 71 percent) females and 20 males, 47 ( 68 percent) white, with ages ranging of 9 to 82 years, and with an avarage disease duration of 62 months. The most frequent diagnoses were Takayasu's arteritis in 13 (19 percent), Buerger's disease in 11 cases (16 percent), poliarteritis nodosa in 7 (10 percent), and cutaneous leucocytoclastic vasculitis in 4. Despite intense and expensive investigation, 15 patients (22 percent) remained without defined diagnose, being classified as nonspecified systemic vasculitis. ${ }^{10}$ Evidently, most patients with manifestations restricted to the skin were attended in the Dermatology Clinic, and not referred to our service.

The treatment of systemic vasculities varies according to the different diagnoses, but to obtain quick control of inflammation, high-dose corticosteroid is used in most cases. Immunosupressive drugs, especially cyclophosphamide, need to be used in all cases of Wegener granulomatosis," microscopic polyangitis, and in cases of polyarteritis nodosa or nonspecific vasculitis with visceral involvement. ${ }^{12}$ In other vasculitis syndromes such as Takayasu's arteritis, temporal arteritis or cutaneous

\section{REFERENCES}

1. Mandel BFR, Hoffman GS. Differentianting the vasculitis. Rheum Dis Clin North Am 1994;20(2):409-42.

2. Lie JT. Vasculitis, 1815 to 1991: Classification and Diagnosis Specificity. J Rheumatology 1992;19(1):83-9.

3. Zeek PM. Periarteritis nodosa: A critical review. Am J Clin Pathol 1952;22:777-90.

4. Gillian JN, Smiley JD. Cutaneous necrotizing vasculitis and related disorders. Ann Allergy 1976; 37:328-39.

5. Fauci AS, Haynes BF, Katz P. The spectrum of vasculitis: clinical, pathologic, immunologic, and therapeutic considerations. Ann Intern Med 1978;89:660-76.

6. Lie JT. Nomenclature and classification of vasculitis: Plus ca change, Plus c'est La meme chose. Arthritis Rheum 1994;37(2):181-86. vasculitis, corticosteroid is the treatment of choice, and only in nonresponsive cases or in the presence of important side effects of corticosteroid therapy should immunosupressive drugs be administered. Unlike in vasculitis affecting medium-size vessels, in cases of Takayasu arteritis ${ }^{13}$ and temporal arteritis, ${ }^{14}$ the immunossupressive drug most used is methotrexate in low doses, once a week. The efficacy of intravenous immunoglobulin (IVIg) is established only for Kawasaki's disease to prevent development of coronary artery aneurysms. ${ }^{15}$ IVIg, ${ }^{16}$ and monoclonal antibodies targeted to $\mathrm{T}$ cells ${ }^{17}$ are alternatives to conventional immunosupressive drugs for the management of systemic vasculitis, but have been used in only a few cases and need to be more extensively studied. Plasmapheresis proved useful in cases of hepatitis B-related polyarteritis nodosa, ${ }^{18}$ but its excessive cost renders it seldom used in Brazil.

Cases not amenable to exact classification or diagnosis need to be treated according to the severity of each case. Thus, patients with visceral involvement should be treated with high-dose corticosteroid and immunosupressive drugs. Platelet antiaggregant or anticoagulant drugs may be useful in cases in which there is a high risk of thrombosis complicating vasculitis, especially in cases affecting medium- or large-sized blood vessels.

We conclude that vasculitic syndromes are difficult to diagnose, but when obtained, the exact diagnosis allows better choice of treatment and knowledge of the prognosis for each specific case.

7. Jennette JC, Falk RJ, Andrassy K, et al. Nomenclature of systemic vasculitides: proposal of an international consensus conference. Arthritis Rheum 1994;37:187-92.

8. Sundy JS, Haynes BF. Pathogenic mechanisms of vessel damage in vasculitis syndromes. Rheum Dis Clin North Am 1995;21(4):861-81.

9. Gross WL. Antineutrophil Cytoplasmic autoantibody testing in vasculitides. Rheum Dis Clin North Am 1995;21(4):987-1011.

10. Sato EI, Sallorenzo C, Hatta F et al. Aspectos clínicos e laboratoriais das vasculites. Rev Bras Reumatologia (abstr) 1996;36(5):321.

11. Hoffman GS, Kerr GS, Leavit RY, et al. Wegener granulomatosis. An analysis of 158 patients. Ann Int Med 1992;116:48-98.

12. Lhote F, Guillevin L. Polyarteritis nodosa, microscopic polyangiitis and Churg-Strauss syndrome. Rheum Dis Clin North Am 1995;21(4):911-47. 
13. Kerr GS. Takayasu's arteritis. Rheum Dis Clin North Am 1995;21(4):1041-58.

14. Nordborg E, Nordborg C, Malmvall Bo-Erikl, Andersson R, Bengtsson, Bengt-Ake. Giant cell arteritis. Rheum Dis Clin North Am 1995;21(4):1013-26.

15. Newburger JW, Takahashi M, Beiser AS, et al. The treatment of Kawasaki syndrome with intravenous gamma globulin. N Engl J Med 1986;315:341-345.
16. Richter C, Schnabel A, Csernok E, et al. Treatment of ANCAassociated systemic vasculitis with high-dose intravenous immunoglobulin. Arthritis Rheum (abstr) 1994;37:(suppl 9) S 353.

17. Lockwood CM, Thiru S, Isaacs JD, et al. Long-term remission of intractable systemic vasculitis with monoclonal antibody therapy. Lancet 1993;341:620-23.

18. Guillevin L, Lhote F, Cohen P, et al. Polyarteritis nodosa related to hepatitis B virus. Medicine 1995;74(5):238-53. 